\title{
Wahlfreiheit und Nutzerrollen im deutschen Bildungssystem
}

\author{
Das deutsche Schulsystem ist hinsichtlich der freien Wahl des Schulorts und Schultyps \\ stark reglementiert. Dennoch existieren Schlupflöcher, die Eltern und Schülern eine \\ gewisse Wahlfreiheit ermöglichen und insbesondere von gebildeten und sozio-ökono- \\ misch bessergestellten Eltern auch genutzt werden. Der Beitrag untersucht, inwieweit \\ sich mit einem solchen aktiven Wahlverhalten auch die zugedachten Rollen der Nutzer \\ des Bildungssystems verändern und welche Rückwirkungen dies gegebenenfalls auf \\ das gesamte Bildungssystem hat.
}

\section{STEPHAN KÖPPE}

\section{Einleitung}

Anders als in Deutschland wird andernorts seit Längerem intensiv darüber diskutiert, wie Schüler und Eltern mehr Wahlfreiheit im Bildungssystem erhalten können. Die zentrale politische Stoßrichtung solcher Debatten ist, dass Nutzer von Bildungseinrichtungen quasi wie Konsumenten von Dienstleistungen ihre Schule selbst auswählen können. ${ }^{\circ}$ Die Schulen würden in der Folge versuchen, möglichst viele Schüler anzuziehen, was nur erreicht würde, wenn sie eine bessere Qualität als ihre direkten Konkurrenten anböten. Insgesamt - so die Hoffnung - solle somit die Qualität aller Schulen verbessert werden. In vielen OECD-Ländern wurde die freie Schulwahl in den letzten 20 Jahren ausgebaut, weshalb Schüler und Eltern neben der staatlichen Gesamtschule nun fristående skolor (freie Schulen) in Schweden, charter schools in den USA oder academies bzw. free schools in England besuchen können. Im internationalen Diskurs erhielt die Schulwahldebatte neuerlichen Auftrieb, als die Organisation für wirtschaftliche Zusammenarbeit und Entwicklung (OECD) in ihrem letzten Bericht „Bildung auf einem Blick“ erstmals Indikatoren zur Schulwahl veröffentlichte (OECD 2010, siehe Indikator D5).

Obwohl die OECD von einer Ausweitung der Wahlfreiheit seit 1985 auch für Deutschland ausgeht (ebd., Tabelle D5.6), ist eine derartige institutionelle Entwicklungsrichtung nicht eindeutig ersichtlich. In Deutschland erfolgt die zentrale Schulentscheidung weiterhin nicht als Auswahl der „besten“ Schule, sondern als Zuweisung der „richtigen“ Schulform im dreigliedrigen Schulsystem. Erst in den letzten fünf Jahren formierte sich sukzessiv eine plurale Bewegung, die mehr Wahlfreiheit im deutschen Schulsystem fordert. Die Fürsprecher sind über religiöse und politische Grenzen hinweg zu finden: sowohl in christlich-konservativen (CDU) und marktliberalen (FDP) Kreisen als auch in linksliberalen Bewegungen (Die Grünen, Paritätischer Wohlfahrtsverband, siehe dazu FDP 2009; Füller 2011; Illich 1971; SZ 2009).

Dieser Beitrag analysiert zum einen, welche Wahloptionen im deutschen Bildungssystem derzeit bestehen und wie die Nutzer hiermit umgehen. Zum zweiten geht es darum zu klären, ob eine erweiterte Wahlfreiheit auch die Nutzerrollen verändert. So weisen beispielsweise in den letzten Jahren neu eingeführte Instrumente wie Kita-Gutscheine (Ott 2010), Hochschulvergleiche (Kamm 2008) oder Bildungsgutscheine für Arbeitsuchende (Kruppe 2009) darauf hin, dass Nutzer von Bildungsdienstleistungen zunehmend auch als aktiv und bewusst auswählende Konsumenten angesprochen werden. Das Augenmerk dieses Beitrages richtet sich gleichwohl ausschließlich auf die Privatschulen

Zur Definition der hier und im Folgenden verwendeten Begriffe „Konsument", „Koproduzent", "Klient" und Bürger vgl. Abschnitt 2. 
der Primar- und Sekundarstufe, weil in diesem Bildungssektor ein komplexes Wechselspiel aus institutionellen Rahmenbedingungen, widerstreitenden Nutzerrollen und sich veränderndem Nutzerverhalten vorzufinden ist.

Meine Ausgangsthese ist, dass der institutionelle Rahmen des deutschen Schulsystems in den vergangenen Jahrzehnten nur geringfügig verändert wurde, weshalb kein Wandel der Nutzerrollen zu erwarten wäre. Das deutsche Schulsystem weist Eltern und Schülern vornehmlich die Rolle des Klienten und des Bürgers zu. Nach Noreisch (2007a, S. 70) bestehen dennoch zahlreiche „Schlupflöcher zur Wahlfreiheit“. Beispielsweise treffen Eltern immer häufiger aktive Entscheidungen bei der Schulwahl und verabschieden sich schrittweise aus dem öffentlichen Bildungssystem. Ohne einen fundamentalen politischen und institutionellen Wandel findet ein inkrementeller, also schleichender Wandel statt (vgl. u. a. Hacker 2005). Das Bildungssystem driftet allmählich hin zu einem konsumentenorientierten System, in dem Privatschulen zunehmend eine größere Rolle spielen. Die Nutzer von Bildungsdienstleistungen werden somit zu aktiven Entscheidern, die zwischen verschiedenen Bildungsangeboten wählen. ${ }^{2}$ Der Beitrag untersucht deshalb, wie die im deutschen Bildungssystem dominierende Klienten- und Bürgerrolle in der letzten Dekade allmählich durch die Rolle des Konsumenten und Koproduzenten ergänzt wurde und welche Folgen dies für das Bildungssystem und seine Zielsetzungen hat.

Zunächst werde ich diskutieren, wer eigentlich die Nutzer von Bildungsdienstleistungen sind und wer die Entscheidung für eine Bildungsinstitution trifft (Abschnitt 2). Im nächsten Schritt werden die Nutzerrollen im öffentlichen Bildungssystem unter Rückgriff auf den einschlägigen Diskussionsstand knapp skizziert (3). Der institutionelle Rahmen für das Wahlverhalten der Nutzer von Privatschulen sowie das tatsächliche Wahlverhalten werden anschließend empirisch untersucht. Dazu verwende ich Daten des Statistischen Bundesamtes und der PISA-Studie 2009 (4). Abschließend werden die sozialen Wirkungen der verschiedenen Nutzerrollen diskutiert und es wird dargestellt, welche politischen Folgen die Abwanderung in den privaten Sektor nach sich ziehen kann (4.4 und 5).

\section{Wer sind die Nutzer und wer trifft die Schulwahl?}

Nutzer von Wohlfahrtsleistungen nehmen komplexe Rollen ein, die im Folgenden als Klienten, Bürger, Koproduzenten und Konsumenten bezeichnet werden (s. Ewert in diesem Heft; Clarke et al. 2007). Die Klientenrolle bezeichnet einen passiven Nutzer, der die Dienstleistungsangebote nicht beeinflusst und die gegebenen Strukturen akzeptiert. Als Bürger nehmen Nutzer aktiv Einfluss auf das Angebot von Wohlfahrtsleistungen, indem sie in demokratischen Ent- scheidungsprozessen ihre Anliegen und Präferenzen einbringen können. Im Vergleich dazu beruht die Konsumentenrolle auf der Möglichkeit, zwischen verschiedenen Dienstleistungen auszuwählen und somit die eigenen Präferenzen durch die Auswahl des bevorzugten Anbieters zu erreichen. Schließlich agieren Nutzer als Koproduzenten, wenn sie bei der Erbringung sozialer Dienstleistungen aktiv beteiligt sind: Schüler tragen beispielsweise maßgeblich zum Lernerfolg bei, wenn sie regelmäßig Hausaufgaben erledigen und sich im Unterricht beteiligen.

Diese vier Rollen sind idealtypische Kategorien, deren Grenzen in der Realität fließend sind. Nutzer können verschiedene Rollen gleichzeitig ausfüllen. Meine Grundannahme ist jedoch, dass einzelne Nutzerrollen in bestimmten Kontexten dominieren bzw. durch politische Reformen oder sozialen Wandel an Gewicht gewinnen oder verlieren. Der institutionelle Rahmen weist den Nutzern bestimmte Rollen zu, diese können von den Nutzern jedoch auch durch nonkonformes Verhalten unterlaufen werden. Im Folgenden konzentriere ich mich vor allem auf diese institutionelle Rollenzuweisung und untersuche, welche Rollen tatsächlich ausgefüllt werden. Die selbst wahrgenommenen Nutzeridentitäten werden in diesem Kontext nicht untersucht.

Im Schulkontext sind die Nutzerrollen auf mehrere Schultern verteilt. Schüler sind die primären Bildungsnutzer in der Primar- und Sekundarstufe. Jedoch im Vergleich $\mathrm{zu}$ anderen Wohlfahrtsnutzern (z. B. Rentenversicherten) sind Schüler besonders in der Primarstufe, aber auch im weiteren Verlauf der Sekundarstufe, keine autonomen Konsumenten. Im Regelfall treffen Eltern die Wahlentscheidungen über die geeignete Schule stellvertretend für ihre Kinder, zumindest bis zu einem bestimmten Alter. Eltern sind somit die Stellvertreterkonsumenten für ihre Kinder. Selbst im Sekundarbereich II werden die Eltern noch als wichtige Ratgeber im Hintergrund die Entscheidung ihrer Kinder beeinflussen, obwohl eine größere Autonomie der Schüler anzunehmen ist.

Diese Trennung von primären Konsumenten (Schülern) und sekundären Entscheidern (Eltern) hat großen Einfluss auf die Konsumentenrolle im Schulbereich. Die Entscheidung für einen Dienstleistungsanbieter wird nur zum Teil von der primären Bildungserfahrung geprägt. Stattdessen wird die Entscheidung der Eltern eher von sekundären Informationen vonseiten der Lehrer, Bekannten oder Medien beeinflusst. Eltern sind jedoch nicht die einzigen Akteure, die Entscheidungen über die Schulwahl treffen. In Deutschland haben auch Lehrer Entscheidungsmacht über die Schulform, die Schüler in der Sekundarstufe besuchen können. Die Schüler sind deshalb zwar die Nutzer der Dienstleistung, aber nicht unbedingt die Letztentscheider über den Bildungsanbieter.

(2) Vgl. in diesem Zusammenhang die britische Debatte in Clarke et al. 2007 und Farrel 2009. 
Gemeinsam füllen Eltern und Schüler eine Bürgerrolle aus, wenn sie die Schulpolitik mitgestalten und sich in Schulgremien engagieren. Schließlich sind Schüler auch Koproduzenten, denn der Lernerfolg hängt auch maßgeblich von der Mitarbeit und dem Engagement der Schüler ab. Eltern sind Koproduzenten, wenn sie beim Unterricht mithelfen, Schulfeste organisieren oder das Schulgebäude renovieren. Wie die Rollen als Klient, Bürger, Koproduzent und Konsument ausgestaltet werden, wird im Folgenden betrachtet.

\section{Klienten und Bürger in öffentlichen Schulen?}

Das staatliche Schulsystem weist den Eltern und Schülern überwiegend eine Klienten- und Bürgerrolle zu. Der Besuch der Grundschule hängt, bis auf einige Ausnahmen, vom Wohnort ab (sogenannte Schulbezirke, vgl. Noreisch 2007a). Die weiterführende Schulform (Haupt-/Realschule, Gymnasium) wird maßgeblich durch die Übergangsempfehlung der Lehrer und Leistung der Schüler beeinflusst (Füssel et al. 2010, S. 93). Eltern und Schüler können in Mitbestimmungsgremien die Schulpolitik beeinflussen (Sacher 2008). Eine Konsumentenrolle wird Eltern und Schülern nicht zugeteilt, weil Schulbezirke oder Schulempfehlungen wenig Wahlfreiheit ermöglichen. Nur mit viel persönlichem Aufwand kann das rigide System umgangen werden.

Allerdings zeigen empirische Studien, dass Eltern die vorhandenen Schlupflöcher im Bildungssystem geschickt nutzen und somit mehr Wahlfreiheiten ausüben als politisch intendiert. Denn trotz der staatlichen Lenkungsfunktion nehmen Eltern und Schüler sich selbst teils als Konsumenten wahr und weichen, wenn sie können, von der staatlichen Zuweisung oder Empfehlung ab. Sie nutzen offizielle (Anträge) und inoffizielle Wege (Scheinadressen), um ihre Kinder an einer anderen Grundschule einzuschreiben (Noreisch 2007a, 2007b, S. 79f.; Riedel et al. 2010, S. 102). Ebenso umgehen sie die Lehrerempfehlung zur weiterführenden Schule (Ditton/Krüsken 2009; Jonkmann et al. 2010). Die zitierten Studien dokumentieren, dass sich zwischen 12 und $35 \%$ der Eltern als Konsumenten verhalten, indem ihre Kinder eine andere als die zugewiesene Schule besuchen. Allerdings: Dieses Wahlverhalten ist nicht gleichmäßig in der Bevölkerung verteilt, denn insbesondere Eltern aus höheren sozialen Schichten und ohne Migrationshintergrund nutzen die vorhandenen Wahloptionen bzw. schaffen sich diese Freiräume durch aktives Handeln (Gresch et al. 2010; Jonkmann et al. 2010; Kristen 2008; Noreisch 2007b; Riedel et al. 2010).

Aber nehmen Eltern auch ihre demokratische Bürgerrolle wahr, die ihnen zugewiesen wird? Und gestalten sie als Koproduzenten aktiv die Schulangebote mit? Laut der PISA-Studie 2009 beteiligten sich 17 \% der Eltern in Schul- gremien. Die Bürgerrolle ist somit vergleichsweise gering, wenn man bedenkt, dass dies die erwartete Nutzerrolle ist. Die Koproduzentenrolle zeigt sich vor allem in extrakurrikularen Aktivitäten (19\%) und bei Unterstützung der Lehrer in der Schule (13\%). Offensichtlich beteiligen sich weniger als $20 \%$ der Eltern an Aktivitäten, von denen die Schule und damit alle Schüler direkt profitieren. Sporadische Unterstützung einzelner Aktivitäten ist dabei noch häufiger als regelmäßige repräsentative Gremienarbeit. Obwohl die institutionellen Rahmenbedingungen die Bürgerrolle fördern, ist sie nicht ausgeprägter als die Konsumentenoder Koproduzentenrolle.

Gleichwohl zeigt die Gesamtschau, dass sich durchaus komplexe Nutzerrollen im öffentlichen Bildungssystem finden. Auch wenn die institutionellen Rahmenbedingungen das Nutzerverhalten im Sinne eines folgsamen Klienten und engagierten Bürgers stark vorprägen, gibt es Rollenalternativen: als aufopferungsvoller Koproduzent oder auch als auswahlorientierter Konsument. Gesellschaftspolitisch relevant ist dabei besonders, dass es - wie bereits erwähnt gerade Eltern aus höheren sozialen Schichten sind, die von der institutionell zugewiesenen Rolle als Klient abweichen und als Konsumenten Schlupflöcher des Systems nutzen, um die Bildungserfahrung ihrer Kinder zu optimieren.

\section{Privatschulen als Alternative im Schulsystem}

Über die institutionellen Rahmenbedingungen und das daraus resultierende Wahlverhalten im deutschen Privatschulbereich ist, im Vergleich zu den öffentlichen Schulen, immer noch wenig bekannt, obwohl Privatschulen zunehmend an Bedeutung gewinnen.

\subsection{Wahloptionen und Zugangsbarrieren}

Das Grundgesetz legt in Artikel 7 den Spielraum für Privatschulen in der Bundesrepublik fest, den die Bundesländer unterschiedlich ausfüllen. ${ }^{\circ}$ In Bezug auf die Schülerund Elternvertretung müssen Privatschulen denselben Regeln folgen wie öffentliche Schulen. Auch in Bezug auf die Schulformen und die Kriterien zum Erreichen eines Abschlusses gelten grundsätzlich dieselben Regeln für private und öffentliche Schulen. Privatschulen dürfen aber von den Aufnahmekriterien der öffentlichen Schulen abweichen, d. h. sie können geringere Kriterien zur Aufnahme in die Gymnasialstufe anlegen (vgl. BVerfG 1969). Eltern können somit die bindende Empfehlung der Lehrer gege-

3 Die Unterschiede in der länderspezifischen Regulierung der Privatschulen sind in KMK 2009 aufgeführt. 
benenfalls umgehen, wenn sie ihre Kinder auf eine Privatschule schicken.

Aus der Nutzerperspektive sind die für den Besuch einer Privatschule anfallenden Schulgebühren eine institutionelle Hürde. Die Privatschulen erhalten zwar öffentliche $\mathrm{Zu}$ schüsse, je nach Bundesland besteht jedoch teilweise eine Finanzierungslücke von bis zu 50 \% der Schülerkosten (Klein 2007, S. 46; KMK 2009). Gleichzeitig können die Eltern $30 \%$ der anfallenden Schulgebühren steuerlich geltend machen bis zu einem Höchstbetrag von $5.000 €$ pro Jahr.

Der Erhebung von Schulgebühren setzt das Grundgesetz enge Grenzen. Das Sondierungsverbot nach den Besitzverhältnissen der Eltern erlaubt praktisch zwei Formen der Gebührengestaltung (vgl. BVerfG 1987): Erstens können Privatschulen eine einheitliche, aber sehr geringe Schulgebühr erheben, die keine Sondierung nach den Besitzverhältnissen bewirkt. Derzeit gilt eine Schulgebühr von rund $100 €$ pro Monat als Obergrenze für diesen Finanzierungstyp (Klein 2007). Meistens haben konfessionelle Privatschulen diese Form der Gebührenordnung und nehmen eher symbolische Beiträge. Zweitens können höhere Schulgebühren verlangt werden, wenn das Einkommen der Eltern bei den Gebühren berücksichtigt wird. Wohlhabendere Eltern bezahlen dann höhere Gebühren als Eltern mit geringem Einkommen. Nur in Rheinland-Pfalz sind Schulgebühren verboten, wenn die Privatschulen öffentliche $\mathrm{Zu}$ schüsse erhalten. Zusätzlich gründen einige Privatschulen Fördervereine, die freiwillige Elternbeiträge einwerben. Diese Spenden können aber quasi-obligatorischen Charakter besitzen und sind möglicherweise eine weitere finanzielle Hürde für die Eltern. Im Rahmen dieser verfassungsrechtlichen Vorgaben variieren die Schulgebühren dennoch stark zwischen den Schulen. Die Finanzierungsregelungen wurden seit dem Bestehen der Bundesrepublik nur geringfügig verändert.

Die Schulgebühren der Privatschulen limitieren den Zugang für Schüler aus Familien mit geringen finanziellen Ressourcen (Eisinger et al. 2010). Aufgrund der Gebührenstruktur ist deshalb zu erwarten, dass vor allem einkommensstarke Eltern die Wahloption der Privatschule für ihre Kinder nutzen. Privatschulen sind zudem eine Alternative für Eltern mit hohen Bildungsaspirationen, wenn der $\mathrm{Zu}$ gang zu einem öffentlichen Gymnasium verwehrt wurde. Und schließlich kann die Pluralität der pädagogischen und weltanschaulichen Bildungsangebote ein Anreiz für die Wahl einer Privatschule sein.

Die institutionelle Konsumentenrolle wird bei dem Besuch einer Privatschule insgesamt gestärkt, weil ein monetäres Vertragsverhältnis geschaffen wird (Schulgebühren) und eine Schule zwischen verschiedenen Angeboten ausgewählt wird. Die Privatschulen sind somit ein weiteres Schlupfloch, um Wahlfreiheit in einem ansonsten stark reglementierten Schulsystem auszuüben. Die Bürgerrolle hingegen, also die Chance, im Rahmen der Schüler- und Elternvertretung partizipativ Einfluss auf die Schulorganisation zu nehmen, unterliegt in öffentlichen und privaten
ABB. 1

\section{Anteil der Privatschulen und Privatschüler in Deutschland 1950-2010}

Angaben in Prozent - Schulen - Schüler aller Schulformen

- Schüler Gymnasium - Schüler Grundschule

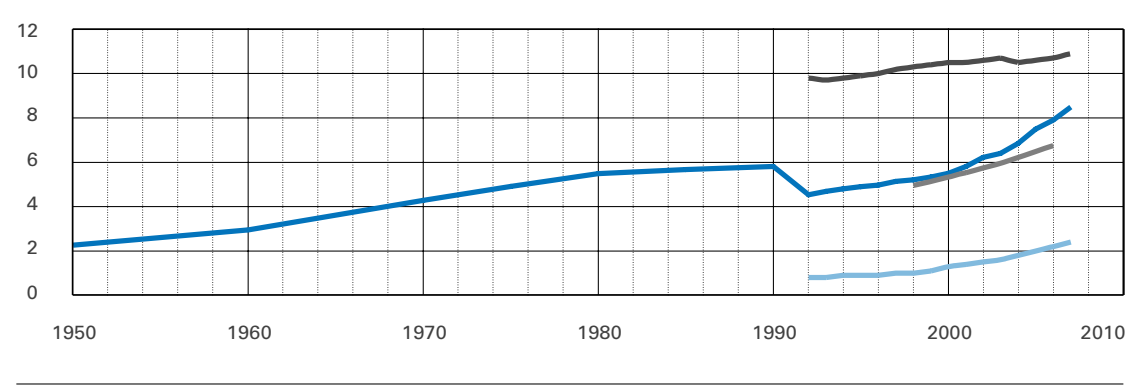

Quelle: Destatis 2000, 2008 (bis 1991 Westdeutschland, ab 1992 Gesamtdeutschland); Berechnungen des Autors.
Schulen denselben Regeln. Somit gibt es diesbezüglich kein institutionelles Unterscheidungskriterium gegenüber öffentlichen Schulen.

\subsection{Privatschulen im Aufwind}

Wie wirken sich diese institutionellen Rahmenbedingungen nun auf die Nutzerrollen aus? Abbildung 1 zeigt einen eindeutigen Trend: Der Anteil der Privatschulen steigt seit nunmehr 60 Jahren kontinuierlich an. Privatschulen erfreuen sich ungebrochenen Zustroms. ${ }^{\circledR}$ Der Anstieg der Privatschülerzahlen bestätigt diesen Trend. Immer mehr Eltern und ihre Kinder wenden sich Privatschulen zu und kehren den öffentlichen Schulen den Rücken. Eine Zunahme der Konsumentenrolle wird auch hier offenkundig. Eltern vergleichen die Bildungsangebote öffentlicher und privater Schulträger und kommen offensichtlich zu dem Schluss, dass Privatschulen eine attraktive Alternative sind - trotz erhöhter Kosten (Schulgeld, Fahrtweg etc.).

Ein zweiter Befund wird aus dem Vergleich der verschiedenen Schulstufen 2008/09 deutlich. Im Grundschulalter ist der Anteil der Privatschüler mit 3,5\% erheblich geringer als im Sekundarbereich I. ${ }^{\ominus}$ Die privaten Gymnasien (Sekundarstufe I und II) haben einen überdurchschnittlich hohen Zulauf mit rund $11 \%$ der Gymnasialschüler im Vergleich zu Realschulen (9\%) und Hauptschulen (3 \%).

(4) Der Einbruch nach der Wiedervereinigung erklärt sich daraus, dass in den neuen Bundesländern vor der Wende praktisch keine Privatschulen existierten und ein privates Schulwesen erst nach 1990 aufgebaut wurde.

5 Der Wert liegt für Haupt- und Realschule, Gymnasien, Gesamtschulen, Waldorfschulen bei $9 \%$, nicht in Abbildung 1 aufgeführt, diese Berechnung basiert auf Destatis 2000, 2008. 
TABELLE 1

Privatschulbesuch in Abhängigkeit sozio-demografischer Variablen

\begin{tabular}{|c|c|c|c|}
\hline & (1) & (2) & (3) \\
\hline Geschlecht (Referenz: männlich) & $\begin{array}{c}0.27 \\
(0.32)\end{array}$ & $\begin{array}{c}0.10 \\
(0.27)\end{array}$ & $\begin{array}{c}0.18 \\
(0.27)\end{array}$ \\
\hline Geschwister & $\begin{array}{c}0.29 \\
(0.22)\end{array}$ & $\begin{array}{l}-0.01 \\
(0.22)\end{array}$ & $\begin{array}{c}0.16 \\
(0.24)\end{array}$ \\
\hline \multicolumn{4}{|l|}{$\begin{array}{l}\text { Gemeindegröße (Referenz: weniger als } \\
\text { 15.000 Einwohner) }\end{array}$} \\
\hline 15.000 bis 100.000 Einwohner & $\begin{array}{c}0.33 \\
(0.96)\end{array}$ & $\begin{array}{c}0.33 \\
(0.90)\end{array}$ & $\begin{array}{c}0.52 \\
(0.78)\end{array}$ \\
\hline mehr als 100.000 Einwohner & $\begin{array}{c}0.45 \\
(1.06)\end{array}$ & $\begin{array}{c}0.64 \\
(1.04)\end{array}$ & $\begin{array}{c}0.71 \\
(0.96)\end{array}$ \\
\hline Migrationshintergrund & $\begin{array}{l}-0.66 \\
(0.66)\end{array}$ & $\begin{array}{l}-0.35 \\
(0.62)\end{array}$ & $\begin{array}{l}-0.90 \\
(0.84)\end{array}$ \\
\hline \multicolumn{4}{|l|}{$\begin{array}{l}\text { Schulbildung der Eltern (Referenz: SEK I } \\
\text { oder niedriger) }\end{array}$} \\
\hline Berufsausbildung & $\begin{array}{c}0.63 \\
(0.70)\end{array}$ & $\begin{array}{c}0.97 \\
(0.83)\end{array}$ & $\begin{array}{c}0.89 \\
(0.78)\end{array}$ \\
\hline Abitur / Fachabitur & $\begin{array}{c}0.76 \\
(0.48)\end{array}$ & $\begin{array}{c}1.09 \\
(0.70)\end{array}$ & $\begin{array}{c}1.24^{*} \\
(0.61)\end{array}$ \\
\hline Berufsakademie & $\begin{array}{c}0.57 \\
(0.67)\end{array}$ & $\begin{array}{c}0.78 \\
(0.84)\end{array}$ & $\begin{array}{c}0.68 \\
(0.81)\end{array}$ \\
\hline Hochschulabschluss & $\begin{array}{c}0.57 \\
(0.53)\end{array}$ & $\begin{array}{c}0.91 \\
(0.75)\end{array}$ & $\begin{array}{c}0.99 \\
(0.69)\end{array}$ \\
\hline sozioökonomischer Status der Eltern (ISEI) & $\begin{array}{l}0.03^{* *} \\
(0.01)\end{array}$ & $\begin{array}{c}0.02 \\
(0.01)\end{array}$ & $\begin{array}{l}0.00 \\
(0.01)\end{array}$ \\
\hline $\begin{array}{l}\text { überdurchschnittliches Haushaltseinkommen } \\
\text { (>40.000 €) }\end{array}$ & & $\begin{array}{l}0.83^{* *} \\
(0.26)\end{array}$ & $\begin{array}{c}0.44 \\
(0.29)\end{array}$ \\
\hline Wohlstand an Konsumgütern & $\begin{array}{l}-0.25 \\
(0.20)\end{array}$ & $\begin{array}{l}-0.20 \\
(0.24)\end{array}$ & $\begin{array}{l}-0.24 \\
(0.23)\end{array}$ \\
\hline \multicolumn{4}{|l|}{$\begin{array}{l}\text { Ausgaben für Bildungsdienstleistungen } \\
\text { (Referenz: nichts) }\end{array}$} \\
\hline $1-499 €$ & & & $\begin{array}{l}-0.30 \\
(0.39)\end{array}$ \\
\hline $500-1.199 €$ & & & $\begin{array}{c}1.16 \\
(0.80)\end{array}$ \\
\hline $1.200-2.399 €$ & & & $\begin{array}{l}2.04^{* *} \\
(0.75)\end{array}$ \\
\hline $2.400-3.599 €$ & & & $\begin{array}{l}2.32^{* *} \\
(0.89)\end{array}$ \\
\hline$>3.600 €$ & & & $\begin{array}{l}3.32 * * * \\
(0.95)\end{array}$ \\
\hline Konstante & $\begin{array}{l}-4.34^{* * *} \\
(1.28)\end{array}$ & $\begin{array}{l}-4.92^{* * *} \\
(1.35)\end{array}$ & $\begin{array}{c}-5.60 * * * \\
(1.15)\end{array}$ \\
\hline $\mathrm{N}$ & 2,668 & 1,952 & 1,924 \\
\hline Wald & 113.28 & 73.35 & 495.79 \\
\hline Pseudo $\mathrm{R}^{2}$ & 0.04 & 0.05 & 0.18 \\
\hline
\end{tabular}

Anmerkungen: Standardfehler in Klammern, Pseudo $\mathrm{R}^{2}$ ist McFadden Signifikanz: $+p<0,1,{ }^{*} p<0,05 * * p<0,01 * * * 0<0,001$

Quelle: Logistische Regression basiert auf PISA 2009, gewichtet; Berechnungen des Autors.
Der Trend eines höheren Privatschüleranteils bei höherem Bildungsniveau ist auch international festzustellen (OECD 2010). Dies könnte ein Indiz dafür sein, dass Privatschüler die in der Hälfte der Bundesländer bindende Übergangsempfehlung umgehen, indem sie ein privates Gymnasium besuchen. Im Grundschulbereich fügen sich Eltern vermutlich eher in ihre Bürgerrolle, weil die Bildungschancen noch nicht so stark von der Schulform determiniert werden. Auffällig ist insgesamt der inkrementelle Wandel des Nutzerverhaltens. Ohne veränderte Anreizstrukturen besuchen schrittweise immer mehr Schüler Privatschulen.

\subsection{Determinanten der Privatschulwahl}

Warum Schüler eine Privatschule besuchen, inwieweit die Präferenzen der Eltern für ihre Wahlentscheidung maßgeblich sind und welche multiplen Nutzerrollen Letztere dabei einnehmen, kann mit den Daten der amtlichen Statistik allerdings nicht untersucht werden. Dies kann nur anhand von Mikrodaten geklärt werden. Beispielsweise weisen Lohmann et al. (2009) mit den Daten des Sozio-oekonomischen Panels (SOEP) nach, dass „Schüler sozio-ökonomisch besser gestellter Eltern eher Privatschulen besuchen“ (ebd., S. 645). Dieser Effekt nahm im Zeitverlauf sogar zu. Mit den SOEP-Daten können jedoch die Präferenzen der Eltern für ihre Wahlentscheidung und das Elternengagement als Einflussfaktoren nicht untersucht werden. Der PISA 2009-Datensatz ermöglicht es, diese Faktoren herauszufiltern, sie mit den sozio-demografischen Charakteristika zu vergleichen und komplexere Nutzerrollen zu identifizieren. Im Folgenden analysiere ich, welche Determinanten Eltern dazu bewegen, die private Option zu wählen und sich vom öffentlichen Bildungssystem zu verabschieden.

Die Datengrundlage bezieht sich auf das deutsche Sample der PISA 2009-Studie. Demnach gehen 5,2 \% der Schüler auf eine Privatschule, die restlichen Schüler besuchen eine öffentliche Schule. Im Vergleich zur amtlichen Statistik sind also weitaus weniger Privatschüler in der PISA-Stichprobe erfasst (vgl. $9 \%$ in vergleichbarer Sekundarstufe [SEK] I). Die Variable Schultyp verwende ich als abhängige Variable, d. h. sie gibt die Wahrscheinlichkeit an, eine Privatschule zu besuchen. ${ }^{\odot}$

\subsubsection{Sozio-demografische Variablen}

Tabelle 1 zeigt, wie verschiedene demografische, geografische und sozio-ökonomische Variablen die Wahrschein-

(6 Die logistische Regression schätzt sowohl die Gewichtung der Schüler als auch die Effekte des geschichteten Selektionsverfahrens. Die verwendeten unabhängigen Variablen haben besonders viele fehlende Werte im Elternfragebogen (rund $38 \%$ ), was die Gesamtstichprobe von 4.701 Fällen stark reduziert. Der Anteil der Privatschulen sinkt weiter $(\sim 4,5 \%, N \sim 135)$ und die Ergebnisse büßen deshalb generell Erklärungskraft ein. 
lichkeit eines Privatschulbesuchs beeinflussen. Trotz der Kontrollvariablen Geschlecht, Geschwister, Gemeindegröße, Migrationshintergrund oder Wohlstand an Konsumgütern (Fernseher, Computer, etc.) haben allein das Bildungsniveau und der sozio-ökonomische Status der Eltern signifikante Auswirkungen auf die Schulwahl. Zugespitzt lässt sich sagen: Die Wohlhabenden und Gebildeten wählen für ihre Kinder Privatschulen.

Wenn die Eltern ein überdurchschnittliches Jahresbruttoeinkommen (mehr als $40.000 €$ ) verdienen, ist es knapp $130 \%$ wahrscheinlicher, dass ihre Kinder eine Privatschule besuchen (Tabelle 1, Modell 2). Da die Einkommensvariable ausdrücklich freiwillig ausgefüllt werden konnte, verringerte sich das Sample erheblich, wodurch die Schätzungen mit der Einkommensvariable an Robustheit verlieren. Wenn das Einkommen der Eltern nicht berücksichtigt wird (Tabelle 1, Modell 1), erreicht der sozio-ökonomische Status (Berufsgruppe) als Vergleichsvariable ebenso hohe Signifikanzwerte. Der sozioökonomische Status steht somit stellvertretend für das Einkommen der Eltern.

In Modell 3 (in Tabelle 1) wurde überprüft, ob die Ausgaben für Bildungsdienstleistungen einen Einfluss auf den Besuch einer Privatschule haben. ${ }^{0}$ Da Privatschulen im Gegensatz zu öffentlichen Schulen Schulgebühren erheben, ist zu erwarten, dass die Ausgaben für Bildungsdienstleistungen einen starken Zusammenhang mit dem Besuch einer Privatschule aufweisen. Die privaten Bildungsausgaben werden ausschließlich in Modell 3 zur Veranschaulichung des statistischen Einflusses von Schulgebühren verwendet. In weiteren Analysen wird auf diese Variable verzichtet, weil Schulgebühren letztlich eher eine Eigenschaft von Privatschulen sind als ein beeinflussender Faktor für den Besuch einer Privatschule. Dennoch verdeutlichen die privaten Bildungsausgaben die Rolle eines zahlenden Konsumenten, weshalb sie zumindest an dieser Stelle aufgeführt werden.

Wenn mehr als $1.200 €$ pro Jahr für Bildungsdienstleistungen ausgegeben werden, erhöht sich die Wahrscheinlichkeit eines Privatschulbesuchs signifikant. Zur Interpretation dieses Ergebnisses hilft ein Blick auf die Bildungsausgaben der Haushalte beim Besuch einer privaten oder öffentlichen Schule. Tabelle 2 zeigt deutlich, dass über $80 \%$ der Eltern private Mittel für die Unterrichtung ihrer Kinder aufwenden. Aus den Daten ist nicht zu entnehmen, ob die Ausgaben für Schulgeld oder privaten Unterricht außerhalb der Schule aufgewendet werden. Es besteht jedoch die berechtigte Annahme, dass die Ausgaben für Bildungsdienstleistungen zu einem großen Teil mit Schulgeld gleichzusetzen sind, wenn das Kind eine Privatschule besucht, und andernfalls etwa für private Nachhilfe ausgegeben werden, wenn das Kind eine öffentliche Schule besucht.

Ein Großteil der Eltern wendet bis zu $499 €$ für extrakurrikularen Unterricht auf, ohne ihr Kind auf eine Privatschule zu schicken. Private Nachhilfe oder auch Musikschulen scheinen eine beliebte Alternative zum Besuch einer Privatschule zu sein. Nichtsdestotrotz zeigt Tabelle 2 auch, dass die meisten Privatschulen offensichtlich weniger als
TABELLE 2

\section{Familiären Ausgaben für Bildungsdienstleistungen pro Jahr}

Angaben in Prozent

\begin{tabular}{|c|c|c|c|}
\hline Schulen & öffentlich & privat & total \\
\hline Keine & 18,43 & 6,85 & 17,91 \\
\hline $1-499 €$ & 59,32 & 23,37 & 57,69 \\
\hline $500-1.199 €$ & 13,45 & 22,94 & 13,88 \\
\hline $1.200-2.399 €$ & 6,23 & 27,24 & 7,18 \\
\hline $2.400-3.599 €$ & 1,68 & 9,99 & 2,06 \\
\hline$>3.600 €$ & 0,89 & 9,61 & 1,28 \\
\hline
\end{tabular}

Quelle: Pisa 2009, gewichtet; Berechnung des Autors.

$1.200 €$ Schulgebühren pro Jahr erheben, denn über $50 \%$ der Eltern von Privatschülern entrichten Schulgebühren unter dieser Grenze. Somit wird verständlich, warum in diesem Bereich keine signifikanten Werte erreicht werden, denn immerhin $13 \%$ der Eltern geben 500 bis $1.200 €$ pro Schuljahr für die Unterrichtung ihrer Kinder aus, wenn sie eine öffentliche Schule besuchen.

Bemerkenswert in diesem Zusammenhang ist, dass das Einkommen der Eltern unter Berücksichtigung der Bildungsausgaben nicht mehr signifikant ist (vgl. Tabelle 1, Modell 3). Daraus kann geschlossen werden, dass Schulgebühren unter $1.200 €$ pro Jahr für viele Familien angemessen sind, weil sich auch unterdurchschnittlich verdienende Haushalte in diesem Fall für Privatschulen entscheiden. Wichtiger wird in diesem Kontext das Bildungsniveau der Eltern. Wenn Eltern bereit sind, Schulgebühren zu bezahlen, haben sie wahrscheinlich Abitur. Mit anderen Worten: bildungsbewusste Eltern finanzieren den Privatschulbesuch, auch wenn sie nur ein unterdurchschnittliches Haushaltseinkommen erzielen. Umgekehrt bedeutet das, dass wohlhabendere Familien mit geringem Bildungsstatus ihre Kinder weiterhin zu einer öffentlichen Schule schicken.

Zwar bestimmt die Höhe der entrichteten Schulgebühren sehr viel genauer die Wahrscheinlichkeit, eine Privatschule zu besuchen als alle sozio-demografischen Variablen zusammen (vgl. Pseudo $\mathrm{R}^{2}$ ), allerdings sind, wie bereits erläutert, Schulgebühren nicht vollständig unabhängig von der abhängigen Variable.

Schließlich zeigt Tabelle 2 auch, dass die volle steuerliche Begünstigung privater Bildungsausgaben (5.000 €) nur für die wenigsten Familien relevant ist. Lediglich weniger als $1,3 \%$ der Familien geben mehr als $3.600 €$ aus.

(7) Zu den Ausgaben für Bildungsdienstleistungen zählen beispielsweise: Schulgeld, Gebühren für Nachhilfeunterricht, private Tutoren oder Musikschulen; ausdrücklich ausgeschlossen sind Ausgaben für Wertgegenstände wie Bücher. 
Privatschulbesuch in Abhängigkeit von Elternpräferenzen und Elternengagement

\begin{tabular}{|c|c|c|}
\hline & (1) & (2) \\
\hline \multicolumn{3}{|l|}{ Elternengagement } \\
\hline Elternvertretung & $\begin{array}{l}-0.12 \\
(0.14)\end{array}$ & \\
\hline Elternarbeit & $\begin{array}{l}1.60^{* *} \\
(0.52)\end{array}$ & \\
\hline extrakurrikulare Aktivitäten & $\begin{array}{c}0.24 \\
(0.24)\end{array}$ & \\
\hline Bibliothek & $\begin{array}{l}-1.02 \\
(0.67)\end{array}$ & \\
\hline Unterrichtshilfe & $\begin{array}{l}-0.19 \\
(0.24)\end{array}$ & \\
\hline Vortrag & $\begin{array}{c}0.21 \\
(0.49)\end{array}$ & \\
\hline \multicolumn{3}{|l|}{ Elternpräferenzen bei der Schulwahl } \\
\hline Wohnortnähe & & $\begin{array}{l}-0.57^{* * *} \\
(0.15)\end{array}$ \\
\hline Reputation & & $\begin{array}{c}0.05 \\
(0.16)\end{array}$ \\
\hline Kursauswahl & & $\begin{array}{c}-0.21+ \\
(0.11)\end{array}$ \\
\hline Weltanschauung & & $\begin{array}{c}0.67^{* *} \\
(0.22)\end{array}$ \\
\hline Pädagogisches Profil & & $\begin{array}{c}0.51 \\
(0.32)\end{array}$ \\
\hline Familienmitglieder & & $\begin{array}{c}-0.02 \\
(0.09)\end{array}$ \\
\hline geringe Kosten & & $\begin{array}{c}-0.20 \\
(0.13)\end{array}$ \\
\hline finanzielle Hilfe & & $\begin{array}{c}-0.26+ \\
(0.13)\end{array}$ \\
\hline aktive/angenehme Lernumgebung & & $\begin{array}{l}0.86^{* * *} \\
(0.17)\end{array}$ \\
\hline schulische Leistung & & $\begin{array}{c}-0.14 \\
(0.14)\end{array}$ \\
\hline sichere Lernumgebung & & $\begin{array}{c}0.03 \\
(0.17)\end{array}$ \\
\hline Konkurrenz in Nachbarschaft & $\begin{array}{c}0.29 \\
(0.26)\end{array}$ & $\begin{array}{c}0.28 \\
(0.26)\end{array}$ \\
\hline Konstante & $\begin{array}{l}-3.40^{* * *} \\
(0.37)\end{array}$ & $\begin{array}{l}-4.68^{* * *} \\
(0.64)\end{array}$ \\
\hline $\mathrm{N}$ & 2,816 & 2,672 \\
\hline Wald & 42.09 & 181.70 \\
\hline Pseudo R² & 0.04 & 0.14 \\
\hline
\end{tabular}

Anmerkungen: Standardfehler in Klammern, Pseudo $\mathrm{R}^{2}$ ist McFadden, Signifikanz: $+p<0,1,{ }^{*} p<0,05 * * 0<0,01 * * * p<0,001$

Quelle: Logistische Regression basiert auf PISA 2009, gewichtet; Berechnungen des Autors.
Nachdem die sozio-demografischen Einflussfaktoren untersucht wurden, bietet der PISA-Datensatz auch die Möglichkeit, das tatsächliche Nutzerverhalten und Präferenzen bei der Schulwahl zu analysieren. Modell 1 (Tabelle 3) zeigt die Wahrscheinlichkeit eines Privatschulbesuchs in Abhängigkeit vom Elternengagement, d. h. das tatsächliche Nutzerverhalten. Die Anpassungsgüte (Wald- und Pseudo- ${ }^{2}$ Statistik) deutet darauf hin, dass keine der Variablen besondere Erklärungskraft besitzt - anders als im Vergleich zu den sozio-ökonomischen Variablen in Tabelle 1. Dennoch können wichtige Ergebnisse aus Tabelle 3 herausgefiltert werden: Die Eltern, die ihre Kinder auf eine Privatschule schicken, beteiligen sich seltener an der Elternvertretung als Eltern an öffentlichen Schulen (negativer Koeffizient), was in diesem Modell jedoch noch nicht bestätigt wird (vgl. aber die Signifikanz dieser Variable in Tabelle 4). Sie verhalten sich also weniger als Bürger, sind aber deutlich aktiver bei Aktivitäten zur Instandsetzung der Schulinfrastruktur (siehe Elternarbeit: 10,8 \% private vs. 3,5\% öffentliche Schulen). Sie treten somit als Koproduzenten in Erscheinung. Sie helfen, die schulische Basisausstattung sicherzustellen, die pädagogischen Aktivitäten überlassen sie den Lehrkräften. Zusätzlich zu den Schulgebühren opfern Eltern also einen erheblichen Anteil ihrer Freizeit, um das Gelingen ihrer Investition zu sichern.

In Modell 2 (Tabelle 3) wurden Eltern befragt, wie wichtig ihnen die aufgelisteten Gründe für die Wahl einer Schule sind. Offensichtlich sind die Elternpräferenzen bessere Indikatoren für die Wahl einer Privatschule als das Elternengagement (vgl. Pseudo- $\mathrm{R}^{2}$ ). Die Präferenzen für Reputation, schulische Leistungen, sichere Lernumgebung, Familienmitglieder an derselben Schule oder geringe Kosten scheinen die Entscheidung für eine Privatschule nicht zu beeinflussen. Präferieren Eltern, dass ihre Kinder bestimmte Kurse und Schulfächer besuchen, entscheiden sich einige Eltern eher für eine öffentliche Schule. Das öffentliche Schulwesen bietet also Alternativen, dennoch ist dieser Zusammenhang relativ schwach ausgebildet. Ist den Eltern das pädagogische Schulprofil wichtig, besteht zwar ein positiver Zusammenhang, der jedoch nicht signifikant ist. Im Gegensatz dazu ist die weltanschauliche Präferenz ein hoch signifikanter Indikator für die Privatschulwahl. Eltern, denen die weltanschauliche Ausrichtung der Schule sehr wichtig ist, schicken ihr Kind auf eine Privatschule. Vermutlich entscheiden sich diese Eltern für eine konfessionelle Privatschule.

Wenn Eltern eine Schule in der Wohnortnähe präferieren, bleiben ihre Kinder eher an öffentlichen Schulen. Die öffentlichen Schulen sind offensichtlich häufiger in der Nachbarschaft lokalisiert und werden deshalb zuerst ausgewählt. Erstaunlich ist in diesem Kontext, dass die Gemeindegröße keinen Einfluss auf die Privatschulwahl hat (Tabelle 1). In Großstädten sind Privatschulen wegen der kürzeren Wege meist einfacher zu erreichen, weshalb die Gemeindegröße ebenfalls einen positiven Einfluss haben könnte (vgl. 
Riedel et al. 2010). Der Schluss liegt nahe, dass Eltern, die eine Schule in der Nähe präferieren, eher ihre Kinder an einer öffentlichen Schule einschreiben und das tatsächliche lokale Angebot keinen Einfluss auf diese Entscheidung hat.

Die Einstellungen über Schulkosten sind ebenfalls wichtige Einflussfaktoren der Privatschulwahl. Eltern, die eine hohe Präferenz für finanzielle Hilfe in Form von Stipendien oder Beihilfen haben, bleiben Privatschulen fern. Die Präferenz für öffentliche finanzielle Unterstützung verringert somit die Wahrscheinlichkeit für den Besuch einer Privatschule. Im Vergleich dazu hat die Präferenz für geringe Schulkosten zwar keinen signifikanten Einfluss, aber auch hier ist ein negativer Einfluss aufgrund des negativen Vorzeichens zu vermuten. Und schließlich werden Privatschulen gewählt, wenn sie ein aktives und angenehmes Lernumfeld bieten können. Eltern, denen dieser Aspekt sehr wichtig ist, entscheiden sich häufiger für eine Privatschule, weil sie eine angenehmere Lernumgebung offenbar nicht in öffentlichen Schulen erwarten.

Ebenso wurden die Eltern gefragt, ob die Schule ihres Kindes mit einer oder mehreren Schulen in der Nachbarschaft konkurriert. Die theoretische Erwartung bei Berücksichtigung dieser Variable ist: Wenn Eltern Schulen in der Nachbarschaft als Konkurrenten registrieren, vergleichen sie vermutlich die verschiedenen Bildungsangebote und agieren als marktsondierende Konsumenten. Allerdings zeigt die Wahrnehmung von Alternativen in keinem Modell in Tabelle 3 einen signifikanten Einfluss auf den Besuch einer Privatschule. Ob Eltern konkurrierende Schulen registrieren oder nicht, hat also keinen Einfluss auf die Wahl einer Privatschule. Die direkte Konkurrenz ist offensichtlich nicht ausschlaggebend für den Besuch einer Privatschule. Einschränkend ist anzumerken, dass die Eltern nicht präzise gefragt wurden, ob die Schule ihres Kindes mit einer Privatschule in der Nachbarschaft konkurriert.

\subsection{Diskussion}

Tabelle 4 fasst die vorherigen Ergebnisse in einem übersichtlichen Modell zusammen. Modell 1 zeigt die Effekte ohne das Einkommen der Eltern, in Modell 2 werden hingegen die Einkommenseffekte berücksichtigt. Die Effekte des Elternengagements und der Elternpräferenzen sind unabhängig von dem Einkommen der Eltern. Hervorzuheben ist aber, dass der negative Effekt des Bürgerengagements in dem optimierten Modell signifikant ist, was die Beobachtung einer geringeren Bürgerrolle (vgl. Abschnitt 3) unterstützt. Eltern, deren Kinder eine Privatschule besuchen, nehmen demnach stärker die Rolle des Koproduzenten ein und engagieren sich seltener als partizipative Bürger in der Schulpolitik.

Die Elternpräferenzen lesen sich wie folgt: Wenn Eltern die schulische Weltanschauung und eine angenehme Lernumgebung wichtig ist, schicken sie ihre Kinder auf eine Privatschule, wovon insbesondere private Konfessionsschulen profitieren. Wenn die Eltern aber eine Schule in der Nachbarschaft präferieren und zusätzliche finanzielle Hilfe be-
TABELLE 4

\section{Privatschulbesuch in Abhängigkeit von Bildung, Einkommen, Engagement und Präferenzen der Eltern}

\begin{tabular}{|c|c|c|}
\hline & (1) & (2) \\
\hline \multicolumn{3}{|l|}{ Schulbildung der Eltern (Referenz: SEK I oder niedriger) } \\
\hline Berufsausbildung & $\begin{array}{r}1.11 * \\
(0.55)\end{array}$ & $\begin{array}{c}0.80 \\
(0.73)\end{array}$ \\
\hline Abitur / Fachabitur & $\begin{array}{l}1.40 * * * \\
(0.42)\end{array}$ & $\begin{array}{c}1.18 \\
(0.73)\end{array}$ \\
\hline Berufsakademie & $\begin{array}{c}0.96 \\
(0.60)\end{array}$ & $\begin{array}{c}0.50 \\
(0.81)\end{array}$ \\
\hline Hochschulabschluss & $\begin{array}{c}1.20^{*} \\
(0.50)\end{array}$ & $\begin{array}{c}0.99 \\
(0.79)\end{array}$ \\
\hline sozioökonomischer Status der Eltern (ISEI) & $\begin{array}{c}0.02+ \\
(0.01)\end{array}$ & $\begin{array}{c}0.01 \\
(0.01)\end{array}$ \\
\hline überdurchschnittliches Haushaltseinkommen (>40 $000 €$ ) & & $\begin{array}{c}0.85^{* *} \\
(0.33)\end{array}$ \\
\hline \multicolumn{3}{|l|}{ Elternengagement } \\
\hline Elternvertretung & $\begin{array}{c}-0.40 * \\
(0.18)\end{array}$ & $\begin{array}{c}-0.65^{*} \\
(0.26)\end{array}$ \\
\hline Elternarbeit & $\begin{array}{r}1.57^{*} \\
(0.67)\end{array}$ & $\begin{array}{r}1.47^{*} \\
(0.59)\end{array}$ \\
\hline \multicolumn{3}{|l|}{ Elternpräferenzen bei der Schulwahl } \\
\hline Wohnortnähe & $\begin{array}{l}-0.75^{* * *} \\
(0.16)\end{array}$ & $\begin{array}{l}-0.68 * * * \\
(0.18)\end{array}$ \\
\hline Weltanschauung & $\begin{array}{l}0.80^{* * * *} \\
(0.14)\end{array}$ & $\begin{array}{l}0.83^{* * *} \\
(0.13)\end{array}$ \\
\hline finanzielle Hilfe & $\begin{array}{c}-0.28^{* *} \\
(0.10)\end{array}$ & $\begin{array}{c}-0.25+ \\
(0.13)\end{array}$ \\
\hline aktive/angenehme Lernumgebung & $\begin{array}{l}0.85^{* * *} \\
(0.15)\end{array}$ & $\begin{array}{l}0.87^{* *} \\
(0.27)\end{array}$ \\
\hline Konstante & $\begin{array}{l}-5.89 * * * \\
(0.62)\end{array}$ & $\begin{array}{l}-6.28 * * * \\
(0.80)\end{array}$ \\
\hline $\mathrm{N}$ & 2,536 & 1,891 \\
\hline Wald & 308.63 & 256.24 \\
\hline Pseudo $\mathrm{R}^{2}$ & 0.18 & 0.18 \\
\hline
\end{tabular}

Anmerkungen: Standardfehler in Klammern, Pseudo $\mathrm{R}^{2}$ ist McFadden, Signifikanz: $+p<0,1,{ }^{*} p<0,05 * * p<0,01 * * * p<0,001$.

Quelle: Logistische Regression basiert auf PISA 2009, gewichtet, Berechnungen des Autors. 
schulbesuch ist umso wahrscheinlicher, wenn die Eltern überdurchschnittlich verdienen. Wenn das Einkommen der Eltern nicht berücksichtigt wird, dann ist das Bildungsniveau der Eltern ausschlaggebend. In diesem Fall wählen die Eltern mit Abitur für ihre Kinder eine Privatschule (Tabelle 4, Modell 1).

Zusammenfassend bleibt festzuhalten, dass Eltern erhebliche persönliche (Elternmitarbeit) und finanzielle (Schulausgaben, Transportkosten) Ressourcen aufwenden, um ihr Kind auf eine Privatschule zu schicken. Eltern präferieren eine Privatschule aus weltanschaulichen Gründen und wegen der angenehmeren Lernumgebung. Zusammengenommen können diese Faktoren erhebliche segregierende Effekte auslösen: Die segregierenden Konfliktlinien verlaufen demnach entlang der finanziellen Ressourcen, der Weltanschauung und des Bildungsniveaus. Da die Religion des Kindes nicht erfasst wurde, kann die religiöse Segregation nicht direkt untersucht werden, sondern nur über die Präferenz für ein weltanschauliches Schulprofil vermutet werden. Der direkte Einkommenseffekt (Einkommen der Eltern) erscheint ebenso nur bedingt robust, weil sich die Fallzahl stark bei Berücksichtigung dieser Variable verringerte. Der indirekte Einkommenseffekt, gemessen an der Präferenz für finanzielle Hilfe, bestätigte die Vermutung allerdings. Das Bildungsniveau der Eltern war in allen Modellen ohne Berücksichtigung des Einkommens sehr wichtig.

Was sagen diese Befunde nun über mögliche Veränderungen der Nutzerrolle im privaten Bildungssektor aus?

(1) Zu konstatieren ist, dass sich Eltern zunehmend als Konsumenten verhalten, weil immer mehr Eltern ihre Kinder auf eine Privatschule schicken. Eltern äußern verstärkt unterschiedliche Bildungspräferenzen und sind bereit, diese Präferenz zu realisieren - selbst wenn sie, wie im Fall der Privatschulen, dafür erhebliche persönliche und finanzielle Mittel aufwenden müssen. Allein die Schulgebühren der Privatschulen stärken die Konsumentenrolle, weil eine Vertragsbeziehung zwischen der Schule und den Schülern bzw. Eltern entsteht. Die Weltanschauung als weiteres wichtiges Motiv ist gleichzeitig ein Indiz für eine Konsumentenrolle, die stark von normativen Idealen geleitet wird. Gleichwohl sind die beobachteten Konsumenten insofern keine „mündigen" Verbraucher, als sie darauf verzichten, verschiedene Schulalternativen zu vergleichen. Die „bewusste“ Wahrnehmung und Prüfung von Alternativen - das zeigt Tabelle 3 - war nicht relevant bei der Wahl einer Privatschule.

(2) Die erstarkte Konsumentenrolle wird dadurch ergänzt, dass Nutzer des Bildungssystems, in diesem Fall Eltern, auch als Koproduzenten agieren (Elternarbeit).

(3) Festzuhalten ist, dass die Bürgerrolle (Gremienarbeit) eher weniger eingenommen wird, wenn die Kinder eine Privatschule besuchen.
Insgesamt bestätigt die Analyse somit, dass Eltern zunehmend als Konsumenten und Koproduzenten im privaten Bildungsmarkt agieren, aber ihre Bürgerrolle dort seltener ausfüllen. Die neuen Rollen als Konsument und Koproduzent haben die alten Rollen - als Klient oder Bürger, die das deutsche Schulsystem Eltern und Schülern primär zuweist noch nicht verdrängt. Öffentliche Schulen können insbesondere von den Privatschulen lernen, wie die Koproduzentenrolle gestärkt werden kann, denn die geopferte Freizeit der Eltern kann eine große Bereicherung für das Schulleben sein.

\section{Schlussfolgerungen}

In diesem Beitrag habe ich gezeigt, wie sich sowohl in der Grund- und Sekundarschule als auch im öffentlichen und privaten Bildungswesen die Nutzer zunehmend als Konsumenten und Koproduzenten verhalten, obwohl ihnen der institutionelle Rahmen des deutschen Schulsystems die Rollen des Klienten und Bürgers zuschreibt. Die Eltern agieren stellvertretend für ihre Kinder und unterlaufen das stark reglementierte System, indem sie die Schlupflöcher zur Wahlfreiheit nutzen. Dabei umgehen sie die ihnen zugewiesenen Schulen auf legale und halblegale Weise und wechseln den Schulbezirk, die Schulform oder zu einer Privatschule.

Solche Wahloptionen werden aber vornehmlich von den höheren sozialen Schichten oder Eltern mit ausreichenden persönlichen und finanziellen Ressourcen genutzt. Damit lassen die Kinder aktiver, wohlhabender und gebildeter Eltern die öffentliche Schule in ihrer Nähe hinter sich und zurück bleiben die Schüler von ressourcenärmeren Eltern. Tendenziell führt das beobachtete Wahlverhalten zu einer sozialen Segregation der Schülerschaft und kann somit ohnehin vorhandene Ungleichheiten der Bildungschancen und -leistungen weiter verstärken. Die soziale Heterogenität der Schulen nimmt ab, was insbesondere die Aufstiegschancen der Schüler aus bildungsfernen Elternhäusern verringert.

Dieser Wandel des Nutzerverhaltens erfolgt inkrementell, ohne dass eine große Schulreform die Anreize für Schüler und Eltern, eine Privatschule zu besuchen, verändert hätte (vgl. Hacker 2005; Mahoney/Thelen 2010). Das veränderte Nutzerverhalten erhöht somit den politischen Druck, das Bildungssystem zu verändern, wenn die ursprünglich intendierten Wirkungen der Schulen - Chancengleichheit und Bildungsgerechtigkeit - weiterhin erreicht werden sollen. Politisch wurde dieser Wandel der Nutzerpräferenzen bisher nur von den kleinen Parteien (FDP, Grüne) oder von einzelnen Verbänden (wie dem Paritätischen Wohlfahrtsverband) thematisiert und problematisiert. Drei politische Szenarien wären als Antwort auf den schleichenden Wandel denkbar. 
(1) Der Status quo bleibt erhalten und die beschriebenen Tendenzen sozialer Ungleichheit, Abwanderung in den Privatschulsektor und verstärkte Konsumenten- und Koproduzentenrolle nehmen zu - mit offenem bildungspolitischen Ausgang.

(2) Die quasi als „Schlupflöcher“ des relativ rigiden Systems vorhandenen Schulwahloptionen für Eltern und Schüler werden abgeschafft, um eine höhere Chancengleichheit zu erreichen. Dies könnte jedoch zu einem öffentlichen Aufschrei wie in Hamburg führen, als geplant wurde, die Gym nasien abzuschaffen. Gerade die politisch einflussreichen höheren sozialen Schichten profitieren von den vorhandenen Wahlmöglichkeiten und könnten gegen diesen Reformpfad politisch mobilisieren.

(3) Der Weg, Wahlfreiheit zu befördern, könnte gezielt und programmatisch eingeschlagen werden. Die Politik würde sich in diesem Szenario dem wachsenden Druck der Nutzer beugen. Die politischen Entscheider stehen damit jedoch vor der Aufgabe, Wahlfreiheit dann auch in einem fairen institutionellen Rahmen zu ermöglichen. Die Anreizstrukturen für die Nutzer und die Anbieter müssten so gestaltet werden, dass segregierende Effekte geringer werden und Begabungen unabhängig von dem familiären Hintergrund gefördert werden (z. B. Thaler/Sunstein 2009, S. 201ff.).

Ungeachtet der politischen Herausforderungen, mehr Chancengleichheit im deutschen Bildungssystem sicherzustellen, produziert das jetzige System aus Zuweisung und Schlupflöchern segregierende Effekte. In diesem Beitrag wurden diese verborgenen Mechanismen aus Zuweisung und Wahlfreiheit im deutschen Bildungssystem analytisch beleuchtet. Eine solche Analyse kann jedoch nur die sozialen Ungleichheiten der Wahlfreiheit aufzeigen und somit Grundlagen für eine politische Entscheidungsfindung legen. Die Änderung des institutionellen Rahmens bleibt letztlich eine politische Aufgabe.

\section{LITERATUR}

Bundesverfassungsgericht (BVerfG) (1969): Beschluss 27, 195 - Anerkannte Privatschulen

Bundesverfassungsgericht (BVerfG) (1987): Beschluss 75, 40 - Privatschulfinanzierung

Clarke, J./Newman, J./Smith, N./Vidler, E./Westmarland, L. (2007): Creating Citizen-Consumers. Changing Publics and Changing Public Services, London Destatis (2000): Bildung und Kultur, Fachserie 11, Reihe S.2, Allgemeinbildende und berufliche Schulen 1950 bis 1999, Stuttgart

Destatis (2008): Bildung und Kultur, Fachserie 11, Reihe 1.1, Private Schulen, Schuljahr 2007/08, Wiesbaden

Ditton, H./Krüsken, J. (2009): Bildungslaufbahnen im differenzierten Schulsystem - Entwicklungsverläufe von Laufbahnempfehlungen und Bildungsaspirationen in der Grundschulzeit, in: Zeitschrift für Erziehungswissenschaft 12 (Sonderheft), S. 74-102

Eisinger, B./Randoll, D./Warndorf, P. K. (2010): Privatschulfinanzierung, in: Barz, H. (Hrsg.): Handbuch Bildungsfinanzierung, Wiesbaden, S. 249-260

Farrell, C.M. (2009): The consumer in education, in: Simmons, R./Powell, M./ Greener, I. (Hrsg.): The consumer in public services. Choice, values and difference, Bristol, S. 119-135

Freie Demokratische Partei (FDP) (2009): Die Mitte stärken. Deutschlandprogramm der Freien Demokratischen Partei, Hannover

Füller, C. (2011): Mit der Bürgerschule Armut bekämpfen, in: TAZ vom 30.03. Füssel, H.-P./Gresch, C./Baumert, J./Maaz, K. (2010): Der institutionelle Kontext von Übergangsentscheidungen: Rechtliche Regelungen und die Schulformwahl am Ende der Grundschulzeit, in: Maaz, K./Baumert, J./Gresch, C./ McElvany, N. (Hrsg.), a.a.O., S. 87-106
Gresch, C./Baumert, J./Maaz, K. (2010): Empfehlungsstatus, Übergangsempfehlung und der Wechsel in die Sekundarstufe I: Bildungsentscheidungen und soziale Ungleichheit, in: Maaz, K./Baumert, J./Gresch, C./McElvany, N. (Hrsg.), a.a.O., S. $201-227$

Hacker, J. S. (2005): Policy Drift: The Hidden Politics of US Welfare State Retrenchment, in: Streeck, W./Thelen, K. (Hrsg.): Beyond Continuity. Institutional Change in Advanced Political Economies, Oxford, S. 40-82

Illich, I. (1971): Deschooling Society, London

Jonkmann, K./Maaz, K./Baumert, J./McElvany, N. (2010): Die Elternentscheidung beim Übergang in die Sekundarstufe I - Eine theoretische Adaption und empirische Überprüfung des Erwartungs-Wert-Modells, in: Maaz, K./Baumert, J./Gresch, C./McElvany, N. (Hrsg.), a.a. O., S. 253-282

Kamm, R. (2008): Wettbewerb in der Hochschulbildung: Hochschulen zwischen Marktzwang und staatlicher Steuerung. Manuskript, vorgestellt auf der DVPWTagung „Die nächste großeTransformation? Marktschaffende Politik: Ursachen, Dynamiken, Ergebnisse", Köln, 4.-5. September

Klein, H.E. (2007): Privatschulen in Deutschland. Regulierung - Finanzierung Wettbewerb, Köln

Kristen, C. (2008): Primary School Choice and Ethnic School Segregation in German Elementary Schools, in: European Sociological Review 24 (4),

S. $495-510$

Kruppe,T. (2009): Bildungsgutscheine in der aktiven Arbeitsmarktpolitik, in: Sozialer Fortschritt 58 (1), S. 9-19

Kultusministerkonferenz (KMK) (2009): Übersicht über die Finanzierung der Privatschulen in den Ländern der Bundesrepublik Deutschland. Zusammenstellung des Sekretariats der Kultusministerkonferenz (Beschluss der Kultusministerkonferenz vom 12.03.2004 i. d. F. vom 09.10.2009), http://www.kmk. org/fileadmin/veroeffentlichungen_beschluesse/2004/2004_03_12-

Privatschulfinanzierung.pdf

Lohmann, H./Spieß, C.K./Feldhaus, C. (2009): DerTrend zur Privatschule geht an bildungsfernen Eltern vorbei, in: Wochenbericht des DIW 76 (38),

S. $640-646$

Maaz, K./Baumert, J./Gresch, C./McElvany, N. (Hrsg.) (2010): Der Übergang von der Grundschule in die weiterführende Schule. Leistungsgerechtigkeit und regionale, soziale und ethnisch-kulturelle Disparitäten, Bonn

Mahoney, J./Thelen, K. (Hrsg.) (2010): Explaining Institutional Change. Ambiguity, Agency, and Power, Cambridge

Noreisch, K. (2007a): Choice as Rule, Exception and Coincidence: Parents' Understandings of Catchment Areas in Berlin, in: Urban Studies 44 (7),

S. $1307-1328$

Noreisch, K. (2007b): School catchment area evasion: the case of Berlin, Germany, in: Journal of Education Policy 22 (1), S. 69-90

Organisation for Economic Co-operation and Development (OECD) (2010): Education at a Glance 2010. OECD Indicators, Paris

Ott, N. (2010): Wer sind die Kunden im Gutscheinsystem?, in: Betz, T./Diller, A./ Rauschenbach (Hrsg.): Kita-Gutscheine. Ein Konzept zwischen Anspruch und Realisierung, Wiesbaden, S. 113-125

Riedel, A./Schneider, K./Schuchart, C./Weishaupt, H. (2010): School Choice in German Primary Schools: How Binding are School Districts?, in: Journal for Educational Research Online 2 (1), S. 94-120

Sacher, W. (2008): Elternarbeit. Gestaltungsmöglichkeiten und Grundlagen für alle Schularten, Bad Heilbrunn

Süddeutsche Zeitung (SZ) (2009): „Wettbewerb fördert Qualität”. Die FDP möchte das Grundgesetz zugunsten freier Schulen ändern, in: Süddeutsche Zeitung vom 02.02 .

Thaler, R.H./Sunstein, C.R. (2009): Nudge. Improving Decisions About Health, Wealth and Happiness, London

\section{AUTOR}

STEPHAN KÖPPE ist Research Fellow an der University of St. Andrews und Visiting Fellow an der University of Edinburgh. Arbeitsschwerpunkte: Wohlfahrtsmärkte, Vergleichende Sozialpolitik.

stephan.koeppe@ed.ac.uk 